\section{Hydralazine-induced MPO-ANCA Renal-Limited Vasculitis Masquerading as Lupus Nephritis}

Sir,

Hydralazine can rarely induce an antineutrophil cytoplasmic antibody (ANCA)-positive vasculitis and less than 80 such cases have been reported hitherto.1,2 Here, we report the case of an elderly patient who was diagnosed with hydralazine-induced ANCA vasculitis after taking hydralazine for 5 years.

A 76-year lady presented with complaints of cough and dyspnea for one day and decreased oral intake for three weeks. She had a past history of diabetes mellitus, hypertension (on hydralazine for two years) and chronic kidney disease (baseline creatinine, $1.9 \mathrm{mg} / \mathrm{dl} 3$ months ago with history of microscopic hematuria for 18 months). Physical examination revealed pallor and decreased air entry at both lung bases. Chest radiograph showed bilateral air-space opacities and CT chest revealed consolidation of the lingula. She was started on antibiotics for pneumonia.

Laboratory investigations were notable for anemia (hemoglobin: $7.9 \mathrm{~g} / \mathrm{dl}$ ) and acute kidney injury (creatinine, $4.0 \mathrm{mg} / \mathrm{dl}$ ). Renal ultrasonography showed bilaterally increased renal parenchymal echogenicity. Urine microscopy showed red cell casts; and urine protein-to-creatinine ratio was consistent with nonnephrotic range proteinuria (300 mg/day). Auto-immune work-up for glomerulonephritis was requested, which was significant for anti-neutrophilantibody (ANA): positive at a dilution of 1:1280 (homogeneous pattern); anti-dsDNA antibody titer: elevated (907 IU/L); ANCA: positive (peri-nuclear pattern); and anti-myeloperoxidase (MPO) antibody titer: elevated (104 AU/mL).

Rest of the auto-immune work-up was within normal limits (Table I). Renal biopsy was performed (Figure 1), which demonstrated a pauci-immune, diffuse necrotizing, crescentic glomerulonephritis.

Based on clinical, serological and pathological findings, a diagnosis of hydralazine-induced MPO-ANCA vasculitis (renal-limited) was made. Hydralazine was stopped immediately and aggressive treatment was initiated with a combination of plasmapheresis and pulse-dose steroids. Plan was to start rituximab therapy as outpatient. Patient's renal function tests had stabilized at the time of discharge and they continued to improve on follow-up.

This patient had evidence of acute kidney injury on top of chronic kidney disease (microscopic hematuria for 18
Table I: Results of auto-immune panel for the patient.

\begin{tabular}{l|l}
\hline Investigation & Result \\
\hline Antinuclear antibody & $\begin{array}{l}\text { Positive at a dilution of 1:1280 with a } \\
\text { homogeneous pattern }\end{array}$ \\
\hline Rheumatoid factor & Negative \\
\hline Complement protein C3 level & Normal \\
\hline Complement protein C4 level & Normal \\
\hline Anti-dsDNA antibody & Titer elevated at $907 \mathrm{IU} / \mathrm{L}$ \\
\hline Anti-Smith antibody & Negative \\
\hline Anti-RNP antibody & Negative \\
\hline ANCA screen & Positive with a peri-nuclear staining pattern \\
\hline Anti-MPO antibody & Titer elevated at $104 \mathrm{AU} / \mathrm{mL}$ \\
\hline Anti-PR3 antibody & Negative \\
\hline Anti-GBM antibody & Negative \\
\hline ASO antibody & Negative \\
\hline Anti-IgA antibody & Negative
\end{tabular}

$\overline{\text { ANCA-antineutrophil cytoplasmic antibody; ASO-anti-streptolysin O; dsDNA-double-stranded }}$ deoxyribonucleic acid; GBM-glomerular basement membrane; IgA-immunoglobulin class A; MPO-myeloperoxidase; PR3-proteinase 3; RNP-ribonuclear protein.

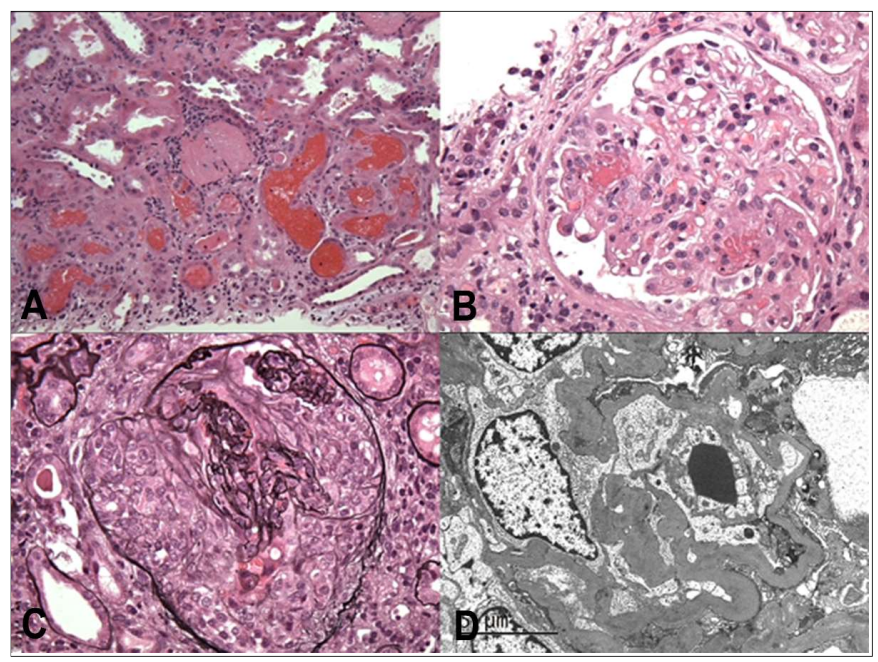

Figure 1: Light microscopic images of renal biopsy specimen demonstrate (A) red cell casts, (B) hypercellular glomerulus with fibrinoid necrosis and (C) crescent formation. (D) Electron micrograph shows extra-capillary proliferation, cellular crescents, mildly thickened glomerular basement membrane and extensive foot process effacement.

months) while taking hydralazine for 2 years. Renal biopsy revealed a pauci-immune crescentic glomerulonephritis. Although an idiopathic ANCA vasculitis could cause similar pathologic findings, the combination of exposure to hydralazine (a drug known to cause MPO-ANCA vasculitis), serologic detection of MPO-ANCA, and elevated ANA and anti-dsDNA antibody titers in the absence of clinical manifestations of lupus were all consistent with hydralazine-induced MPO-ANCA vasculitis. ${ }^{3}$ Priming of neutrophils, for instance, due to an infection, is known to exacerbate ANCA-mediated vasculitides. 4 In this case, the patient had evidence of pneumonia, which may have exacerbated an ongoing vasculitic process. Elevated anti-dsDNA antibody titers were observed in this case, which are uncommonly seen in hydralazine-induced ANCA vasculitis. ${ }^{2}$ This case 
demonstrates that hydralazine-induced MPO-ANCA vasculitis can present in an indolent manner after a long period of latency and with concomitant elevations of ANA and anti-dsDNA antibody titers. Recognition of this peculiar clinical entity is important to avoid a misdiagnosis of idiopathic lupus nephritis, discontinue hydralazine promptly and institute appropriate treatment of a severe, organ-threatening vasculitis.

\section{REFERENCES}

1. Sangala N, Lee RW, Horsfield C, Goldsmith DJ. Combined ANCA-associated vasculitis and lupus syndrome following prolonged use of hydralazine: a timely reminder of an old foe. Int Urol Nephrol 2010; 42:503-6.

2. Yokogawa N, Vivino FB. Hydralazine-induced autoimmune disease: comparison to idiopathic lupus and ANCA-positive vasculitis. Mod Rheumatol 2009; 19:338.

3. Dobre M, Wish J, Negrea L. Hydralazine-induced ANCA- positive pauci-immune glomerulonephritis: a case report and literature review. Renal Fail 2009; 31:745-8.

4. Jennette JC, Falk RJ, Gasim AH. Pathogenesis of ANCA vasculitis. Curr Opin Nephrol Hypertens 2011; 20:263-70.

Noor Ul-Ain Baloch ${ }^{1}$ and Adaeze N. Ibeabuchi ${ }^{2}$

1 Department of Medicine, Rutgers - New Jersey Medical School, University Hospital, 150 Bergen St, Newark, NJ 07103, United States

2 Department of Medicine, Hackensack University Medical Center, 30 Prospect Ave, Hackensack, NJ 07601, United States

Correspondence: Dr. Noor Ul-Ain Baloch, Department of Medicine, Rutgers - New Jersey Medical School, University Hospital, 150 Bergen St, Newark, NJ 07103, United States of America

E-mail: noor2000_2004@hotmail.com

Received: May 26, 2018; Accepted: August 02, 2018 